\title{
Hiperbilirrubinemia no Hemolítica Congénita
}

\author{
Drs. Victor Casanueva E. ${ }^{1}$, Oscar Marín S. ${ }^{2}$, Carlos Calvo M. ${ }^{3}$, \\ Ximena Cid C.4, Mónica Muñoz. 0.1.

\section{Congenital Nonhemolytic Hiperbilirubinemia}

Two cases of Congenitat nonhemolytic lyperbilirubinemia ase reported. Enzymatic assays and clectronic microscopy confirned Crigler-Najjar sind come type I and Il respectively. A brief review of Non hemoly tic hyperbilirubiremias is done.

A pesar, que el término Ictericia sugiere de inmediato enfermedad hepática o hematológica, existe un grupo de alteraciones específicas y familiares en que la hiperbilimubinemia es resultado de una alteración de una función altamente especiali-

1 Depto. de Pediatría, Facultad de Medicina, Universidad de Concepción

2 Depto. de Biología Molecular, Facultad de Ciencias Biológicas y de Recursos Naturales Universidad de Concepción.

3 Depto. de Bioquimica Aplicada, Facultad de Farmacia Universidad de Concepción.

4 Laboratorio Central Hospital Guiliermo Grant Benavente de Concepción. zada en una de las etapas intrahepáticas de la excreción de la bilirrubina.

El Sindrome de Crigler-Najjar pertenece a este grupo de hiperbilirrubinemia no hemolítica congénita de la cual han sido publicadas hasta este momento no más de 80 casos en la literatura mundial.

Se presentan dos casos de niños con hiperbilirrubinemia no conjugada desde recién nacido, cuya causa constituyó injicialmente un problema diagnóstico.

La actividad de la enzima Uridin-difosfo-glucoronil-transterasa (UDPGT) desde una muestra de tejido hepático obtenido por punción percutánea. Para este efecto se separaron los microsomas 
conteniendo la enzima por centrifugación diferencial del homogenizado de hígado en KCL $0,15 \mathrm{M}$ y se incubaron en Tampón Tris $1 \mathrm{M}$, ph 7.6; bilirrubi. na-albúmina $0.51 \mathrm{mM}$ UDPGA $33,5 \mathrm{mM} \quad y$ $\mathrm{MgCL}_{2} 50 \mathrm{mM}$. La actividad se expresó en $\mu \mathrm{g}$ de bilirrubina conjugada producida por gramo de tejido en 30 minutos de incubación.

\section{Casos Clínicos}

\section{L.J.M.}

Nacido el 12 de Diciembre 1980, peso: 2950 gramos al nacer parto de têrmino eutócico. Madre: 39 antos, sana, dos hijos naciđos vivos y muertos en periodo de R.N. con ictericia. Padre: 51 años, sano. Relación parentesco: Abuelo paterno y Abuelo materno eran primos hermanos.

Exámen físico normal al nacer. Al tercer dia se constató ictericia con bilirrubinemia total de 17.6 mg.\% que a los 11 días alcanza a $22,8 \mathrm{mg} . \%$. Se practica recambio sanguíneo con lo cual disminuye a $9 \mathrm{mg} . \%$. Posteriormente persisten valores elevados a expensas siempre de bilirrubina indirecta a pesar de fototerapia mantenida.

Los exámenes de laboratorio descartan procesos hematológicos u otros que expliquen los valores de ésta.

Se inició terapia con fenobarbital a la edad de 3 meses lográndose remisión importante de la bilirrubina sérica.

Se practicó biopsia hepática para estudio de microscopía de luz electrónica ${ }^{1-2} y$ evaluación de actividad de UDP-glucuronil transferase (UDPGT). El análisis de los cortes semifinos (400 $\mathrm{nm}$ ) observados con el microscopio de luz no reveló alteraciones. $\mathrm{El}$ análisis con el microscopio electrónico muestra cambios estructurales en e] hepatocito.

El retículo endoplásmico granuloso (REG) se presenta normal $y$ es posible observar ribosomas adheridos a su superficie.

Los hepatocitos presentan un núcleo grande redondo $y$ central con cromatina muy fina $y$ homogénea y un nucléolo muy bien diferenciado. Otros núcleos eran grandes y regulares y la cromatina más contrastada y formando grumos de diferente densidad (Fig. 1). Había gran desarrollo del retículo endoplásmico liso que ocupaba grandes áreas de citoplasma, formado por una gran cantidad de vesiculas de borde redondeado y de tamaño muy parejo, dispuestas muy cerca una de otra dando a esta zona un aspecto reticulado. La mayor parte de ellas sin contenido en su interior (Fig. 2). Estas zonis del REL excluyen todo otro componente citoplasmático. En otras zonas fue posible observar cisternas de REG en estrecha relación con mitocondrias en las cercanías del núcleo (Fig. 1).

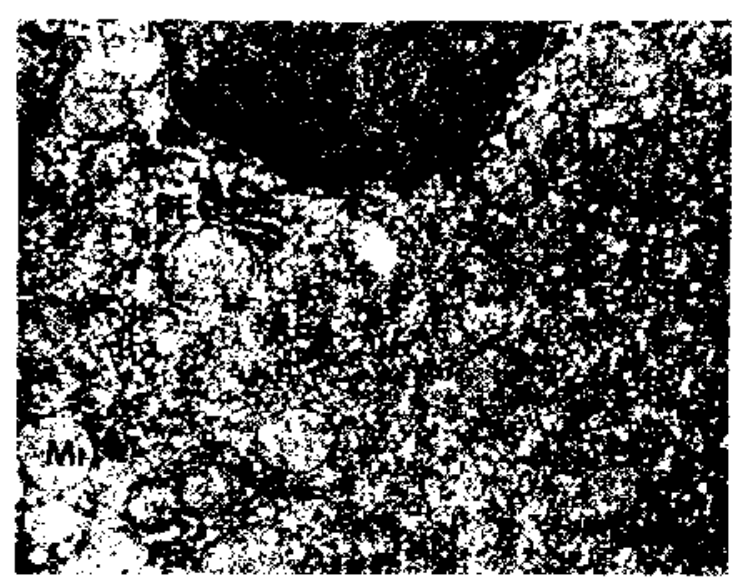

Figura 1.

Región de citoplasma de un hepatocito de L.J.M. mostrando extensas zonas ocupadas por reticulo endoplásmico liso (REL) se observa además núcleo $(\mathrm{N})$, reticulo enđoplásmico granuloso (REG) y numerosas mitocodrias, (mi). $9.400 \mathrm{X}$.

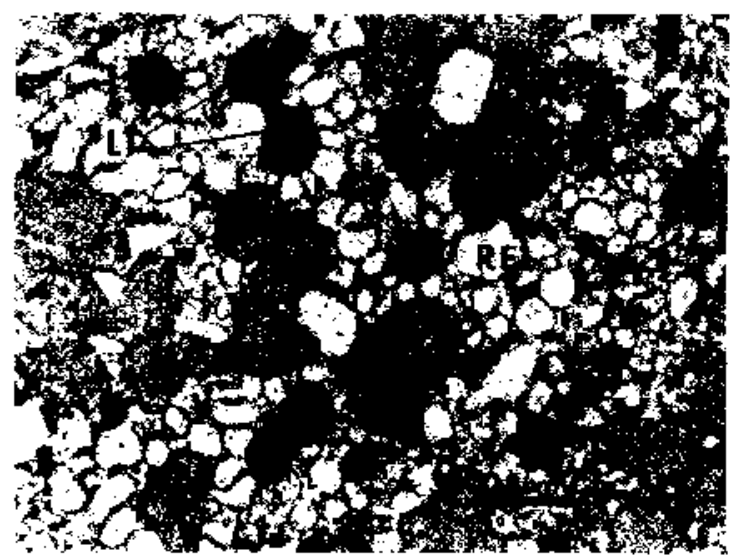

Figura 2.

Hepatocito de LJ.M. se observa el gran desarrollo del retículo endoplásmico liso (REL), además de lisosomas (Li) y mitocondrias (Mi). 23.600 X.

Los bordes de los hepatocitos presentaban canalículos biliares muy dilatados (Fig. 3), con gran cantidad de microvellosidades en su interior y fue posible apreciar en sus límites presencia de desmo. somas.

La medición de la actividad de la enzima UDP-Glucuronil transferasa (UDPGT) mostró un valor de $143 \mu \mathrm{g}$. de bilirrubina conjugada $/ 30 \mathrm{mi}-$ nutos/gramo de tejido hepático. (Valor normal $500 \pm 150 \mu \mathrm{g} \mathrm{x} 30 \mathrm{~min}$. $\mathrm{xgr}$. de tejido).

\section{M.V.G.}

Nacido el 7 de Septiembre de 1978, peso: 2860 gramos al nacer. Madre de 16 años, sana, primigesta, embarazo controlado sin problemas salvo 


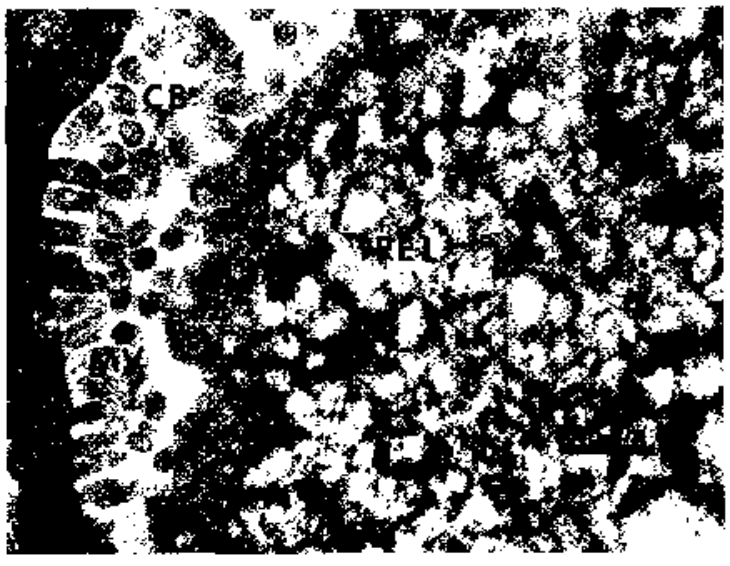

Figura 3.

Borde de un hepalocito de L.J.M. mostrando un canaliculo biliat dilatado(CB) con numerosa microvellosidades (Mv). REL (retículo endoplásmico liso). $28.400 \mathrm{X}$.

pre--eclampsia leve. Padre de 36 años, anteceden. tes de colecistitis. Aparentemente sin relación de consanguinidad entre ellos.

El examen físico al nacer era normal, a los 7 días había ictericia en cara, tronco y extremidades, hasta palmas y plantas con valores séricos de 19 mg.\% de bilirrubina total.

Hígado $2 \mathrm{~cm}$. bajo el reborde costal, orina clara, deposiciones normales. Examen neurológico normal.

La clínica y el laboratorio descartan proceso hemolítico, obstrucción de la vía biliar u anomalias de la bilirrubina conjugada.

La ictericia se ha mantenido sin remisión hasta su último control, a expensas exclusivamente de bilimubina libre a pesar de la terapia mantenida con fenobarbital.

Desde la edad de tres aftos y medio en el examen neurológico existe hipotonia generalizada, reflejos profundos vivos con discreto aumento del área reflexógena y retardo psicomotor importante con edad motora \pm 10 meses y edad psíquica \pm 18 meses.

Se practicó biopsia hepática para estudio en micoscopia de luz y electrónica y evaluación de la actividad de UDP-Glucuronil Transferasa (UDPGT).

El análisis de los cortes semifinos $(400 \mathrm{~nm})$ observando al microscopio de luz no reveló altera. ciones.

El análisis al microscopio electrónico muestra retículo endoplásmico granuloso (REG) normal; adheridos a su superficie, es posible observat ribosomas.

La forma de los hepatocitos se mantiene conservada y presentan un núcleo redondo central y en el citoplasma mitocondrias que ocupan un alto por- centaje de éste. (Fig. 4). Se observan lisosomas en cantidad normal y algunos gránulos de lipofucsina. El reticulo endoplásmico liso (REL) aparece ligeramente hipertrofiado presentando algunas cisternas alargadas pero en su mayoría vesículas de contornos redondeados. Se observan también gránulos de glicógeno distribuidos homogéneamente en todo el citoplasma. En los bordes de los hepatocitos fue posible apreciar canalículos biliares poco dilatados y con microvellosidades en su interior. (Fig. 4).

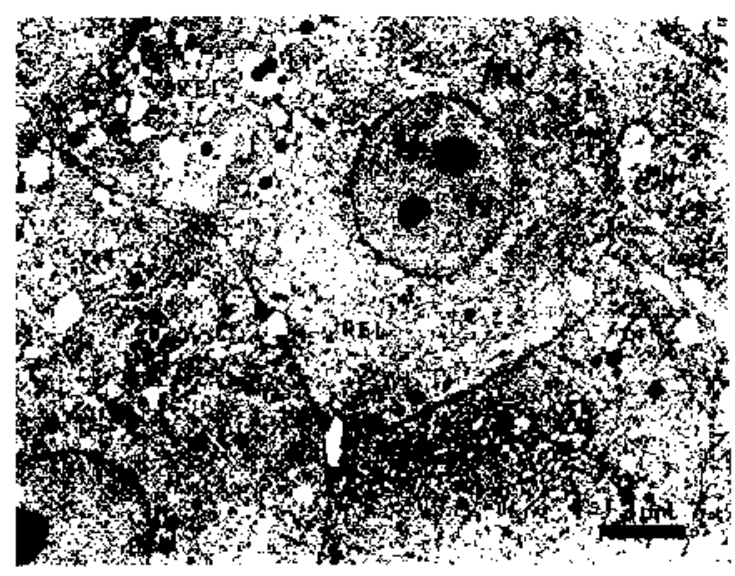

Figura 4.

Hepatocito de M.V.G. Se observa el núcleo redondo y central (N) y numerosas mitocondrias (Mi). Aparecen además nucleolo ( $\mathrm{Nu}$ ), retículo endoplásmico granuloso (REG) y liso (REL), lisosomas ( $\mathrm{Li}$ y y canalículos biliafes (CB). $4.600 \mathrm{X}$.

La medición de la actividad de la enzima UDP-Glucuronil Transferasa (UDPGT) mostró un valor de $0 \mu \mathrm{g}$ de bilirrubina conjugada/ 30 minutos/gramo de tejido hepático.

Controles posteriores efectuados en los últimos 2 años de la biopsia de MVG que no han mostrado variaciones con la terapia efectuada.

\section{DISCUSION}

En 1952 Crigler y Najjar ${ }^{3}$ describieron 7 niños en tres familias emparentadas que tenian ictericia severa no hemolítica dentro de los primeros djas de vida. La ictericia se caracterizaba por un aumento en la sangre de la bilirrubina No Conjugada y su duración era de por vida.

Solamente 2 de estos niños llegaron a la pubertad sin síntomas neurológicos. Eran primos por partida doble. El varón desarrolló daño neurológico a los 16 años y murió de Kernicterus. La niña se desarrolló normalmente hasta los 20 años que comenzó a presentar convulsiones, disartria.

En ninguno de estos pacientes hubo evidencia de hemólisis o de incompatiblidad de grupos clásicos. El estudio histológico del hígado y las prue- 
bas corrientes de función hepática mostraron resultados normales. La bilirrubina plasmática fluccuaba entre 17 y $45 \mathrm{mg}$ \% sin bilirrubinuria.

Los pocos sobrevivientes de este síndrome mueren de Kernicterus en la adolescencia tardia a pesar del empleo de medidas como plasmaféresis, exsanguíneo transfusión y fototerapia. No tienen explicación los casos de 50 años sin Kemictenus ${ }^{5}$.

$A$ rias ${ }^{4}$ diferencia varias alteraciones en que la conjugación está defectuosa o inhibida. Entre cllas el Crigler Najjar I, Crigler Najjar II, homologado al antiguo sindrome de G ilbert.

Los casos clínicos incluidos en esta presentación pertenecerían a Crigler Najjar I (MVG) y Crigler Najjar II (LJV) según la evolución clinica, estudio de la enzima LDP Glucuronil Transferasd (UDPGT) y microscopia electrónica y respuesta frente al inductor enzimático fenobarbital.

En los dos niños comunicados en este trabajo el estudio se hizo después de iniciado el tratamiento con fenobarbital.

Para una mejor comprensión de la etiopatogenia de estas alteraciones revisaremos brevemente el transporte y conjugación normal de la bilirrubina. (Fig. 5).

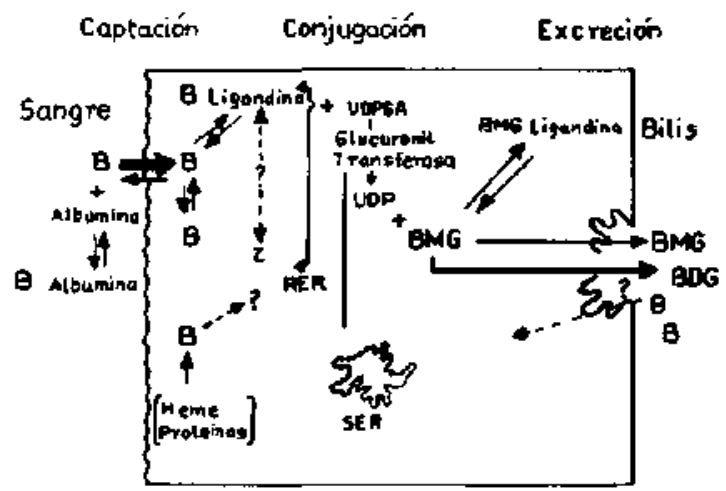

Sigura 5.

Metabolismo hepatico de la bilirrubina: B: bilitrubina, BMG: bilirrubina monoglacuronido, BDG: bilirrubina diglucuronido, UDPG A: Acido uridín difosfoglucurónido. RER: Ietículo endoplástnico rugoso, SI:R: sistema retícuto endoplásmico.

La bilirrubina, derivada del glóbulo rojo senescente secuestrado en las células fagocitarias, es transportada en el plasma unida a la Albúmina y menos de un $10 \%$ se une al glóbulo rojo ${ }^{6}$.

La captación en el hígado es posterior a la separación de la albúmina y la bilirrubina en la membrana plasmática del sinusoide, a través de un transportador, que es compartido por una variedad de aniones orgánicos excluyendo los ácidos biliares $^{7-8}$

El flujo de la bilirrubina a través de la membra- na plasmática es bidireccional y en la ictericia no hemolítica congénita el reflujo de la bilirtubina desde el hepatocito al plasma está aumentado ${ }^{9}$.

Existen varias proteínas en el citosol hepático que unen la bilirrubina en forma reversible. La más importante es la proteina $Y$ (ligandina) que existe solamente en la célula hepática; la proteina $Z$, existe en un menor porcentaje y se encuentra también en el intestino delgado. Esta última capta la bilirrubina sólo cuando la concentración de proteina $Y$, llega a un nivel critico ${ }^{10}$. Se especula si ambas proteinas juegan un rol esencial en ef transporte de la bilirrubina o solatmente sirven para acumular el pigmento en la célula en una forma no tóxica ${ }^{11}$. Una opinión reciente sugiereque forman un sistema de transporte en un medio acuoso, regulando el metabolismo de la bilirrubina ${ }^{12}$. Otros $^{13}$ consideran que la ligandina es decisiva en la captación hepática de la bilirrubina.

En las células hepáticas existe un estanco de bilirrubina monoglucurónido en cuya formación participa solamente la enzima UDPGT (UDPG glucurónico glucuronil transferasa). Esta enzima de microsoma del retículo endoplásmico liso cataliza el transporte del ácido glucurónico desde el UDP - ácido glucurónico a las agliconas ${ }^{14}$. Se cree que el UDPGA se sintetiza en el citoplasma y se encuentra con la enzima situada en la cisterna de la membrana del microsoma por acción de una permeasa 15 .

En la bilis la bilirrubina es predominantemente diglucurónido. la conversión de bilirrubina monoglucurónico a bilirrubina diglucurónido no requiere de la enzima glucuronil transferasa ni del UDPGA. Se ha descrito una segunda enzima en la membrana adyacente al conducto biliar; la Bilirru. bina Glucuronósido-Glucuronosil transferasa (E.C. 2.4.1.9.5.) ${ }^{16}$. Dos moles de bilirrubina monoglucurónido se transforman estequiométrica. mente en un mol de bilirrubina dighucurónido. Inmediatamente de sintetizado es excretado al canalículo biliar, y por lo tanto no se acumula en el hepatocito. El destino de la bilirrubina no conjugada es desconocido. La bilirrubina monoglucurónido aparece en la sangre cuando la velocidad de formación excede de la capacidad excretora de la célula hepática. El điglucurónido se encuentra en el plasma solamente en casos de obstrucción de las vias biliares.

Refiriéndonos al grupo de las hiperbilirrubinemias no hemolíticas crónicas, a pesar de los avances en el conocimiento de su patógenia queda mucho por aclarar todavía.

El sindrome de Crigler Najjar (CN), con falla hepática de conjugación de la bilirrubina, fue clasificada en dos grupos ${ }^{4}$. Esto es artificial pues hay nexos entre el síndrome Gilbert y el C.N.II. El tipo 
de herencia es discutible ${ }^{18}$. Hay casos de C.N.II en adultos $\sin$ respuestas al fenobarbital ${ }^{19}$. La proporción de bilirrubina-monoglucurónido aumenta en la bilis progresivamente desde los controles hasta los pacientes con S. Gilbert, C.N. II y C.N.J ${ }^{20}$. La consanguinidad está presente, como en uno de nuestros casos. Las familiares de los siete primeros casos de C.N. presentaban además homocistinuria, S. de Morquio, enanismo y otros trastornos $21-22$. Algunos autores no encuentran antecedentes de consanguinidad, como ocurrió en el caso de M.V.G. En el tratamiento del C.N. se han empleado dieta hipergrasa, Acido Aspártico ${ }^{23}$ Colestiramina ${ }^{24}$ Fototerapia ${ }^{25}$ sin resultado. Solamente el fenobarbital produce descenso de la bilrrubina en el C.N. II y en el S. Gilbert después de dos semanas de tratamiento ${ }^{26}$. La droga aumenta también el flujo de la bilis, de las sales biliares y fosfolípidos, la actividad de la UDPGT y de la ligandina ${ }^{27-28}$, y produce hipertrofia del aparato de Golgi y proliferación del R.E.L. ${ }^{29-30}$

El síndrome Gilbert presenta una hiperbilirrubinemia fundamentalmente no conjugada. Se hereda de modo autosómico dominante, aumenta durante el ayuno ${ }^{31}$. Su curso es benigno y no se justifica tratarlo con fenobarbital ${ }^{32}$. Existe una reducción de la actividad de la glucuronil transferasa $\sin$ correlación con el nivel de bilirrubina ${ }^{33}$, asociada a un retardo en la acetilación de drogas y la depuración de tolbutamida, sustancia excretada sin glucuronización ${ }^{34}$.

El sindrome de Dubin Johnson es una ictericia benigna, familiar crónica o intermitente relacionada con una falla de excreción canalicular de moléculas orgánicas como Bilirubina, Porfirinas y Agentes Colecistográficos. El transporte de ácidos biljares es normal; la hiperbilirrubinemia es de predominio conjugado ${ }^{35}$. Existe un depósito de melanina en el hepatocito. La excreción urinaria de coproporfirinas I está aumentada y la de copro. porfirina III disminuida. El fenobarbital reduce la ictericia pero el efecto es moderado y sin valor terapéutico ${ }^{36}$.

El S. de Rotor es una hiperbilirrubinemia crónica de predominio conjugado, benigna, familiar, sin pigmentación hepática. La falla sería un defecto en la captación y almacenamiento de aniones orgáni$\cos ^{37}$. Esto es paradojal. Una explicación sería que hay un aumento en el reflujo del hígado al plasma por mala fijación intrahepática de bilirrubina. Esto se ha demostrado en la oveja mutante Southdown, con iguales anomalías. En ella el contenido intrahepático de ligandina es normal ${ }^{38}$. Se confirma ésto por el tratamiento con fenobarbital que no influencia la hiperbilirrubinemia ni la excreción de bromosulfaleina en estos pacientes ${ }^{20}$.

\section{RESUMEN}

Se presentan dos casos de niños portadores de síndrome de Crigler Najjar. Su diagnóstico ha sido confirmado por estudio enzimático y de microscopia electrónica. Se clasifican en tipo I y II de acuerdo con su respuesta al tratamiento con fenobarbital.

Se revisan brevemente las hiperbilirubinemias no hemolíticas congénitas, destacando que a pesar del conocimiento actual queda aún mucho por investigar.

\section{REFERENCIAS}

l Karmovsky $\boldsymbol{H J}$. Fomaldehyde glutaraldehyde fixative of high osmolality for use in electron microscopy $\mathbf{J}$. Cell Biol. 27: $137 \mathrm{~A}, 1965$.

2 Reynolds E.S. The use of $\mathrm{Pb}$ citrate at high $\mathrm{pH}$ as an electron-opaque stain for electron microscopy. J. Cell Biol. 17: 208, 1963.

3 Crigler J.F., Najjar V.A.: Congenital familial nonhemolytic jaundice with kernicterus. Pcdiatrics 10: $169-180,1952$.

4 Arias Irivin $M$. ct als.: Chronic nonhemolytic Linconjugated hyperbilitrubinemia with Glucutanyl transferasc deficiency. Am. J. of Mcd. 47: 395-406, 1969.

5 Gollon J.C. S.N. Haang et als.: Prolonged Survival in 3 brothers with severe type 2 Crigler-Najjar syndrome. Gastroenterology 68: 1543-1555, 1975 .

6 Bornhert, J,L. and Clorenburg, R. Binding of bilirubin to erythrocy tes Proc. Soc. Fxp. Biol. and Med. 142: 1 101.. 1103, 1973.

7 Goresky, C.A. cinado por B.H. Billing Gut: 19: 481-491, 1978 .

${ }^{8}$ Scharschmidt, B.F. and Schmid, R. The micellar sink. Gastroenterology 72:1182, 1977.

9 Biling B, et als.: Defects in hepatic transport of bilirubin Clin. Sc. 27: 245-257, 1964.

10 Levi, A. Gaitman $Z$ and Arias 1. Two Hepatic Cytoplasmic protein Fractions $Y$ and Z.J. of Clin. lnvestigation 48: 2155-2167, 1969.

11 Fleischner, G. and Arias I.M. Structure and Function of Ligandin y protein. GSH Transferase B. and protein in the liver: A progress rcport. Progress in Liver Discase, 5 : $172-182,1976$.

12 Meumissen, J.A.: Ketterer and Heirwegh $\mathrm{K}$. Role of soluble binding proteins in overall hepatic transport of bilitubin. Chemistry and Pliysiology of Bile Pigments, pp. 323-327. Edited by P.D. Berk and N.I. Berlin, 1976 .

13 Wolkoff A.W. Sellin $J$, et als.: Role of the ligandin in trensfe of bilirubin from plasma into the liver. Gastroenterology $73: 1255,1977$.

14 Billing $B$. Twenty five years of progress in bilitubin metabolism. Gut. 19: 481, 1978.

I 5 Berry $C$. and Hallinan $T$. Summary of a novel three component model for UDPG transterase Bioch. Soc. Trans. 4; $650-652,1976$.

16 Wolkoff $A$. $W$. Crigler Najjar syndrome (type I) in an Adult Male. Gastroenterology 76: 840-848, 1979.

17 Mc Kusic Victor. Human Generics. J, of Heredity 71 : $370,1980$.

18 Kreek M. and Steisenger M.H. Reduction of serum Unconjugated bilirubin with phenobarbitone in adult Congerinel nonhemolytic Hyperbilirubinemje. Lancet 2: $73-78,1968$. 
19 Gollon J.L. SN. Huong at als.: Prolonged survival in 3 brothers with severe type 2 Crigler Najjar syndrome. Gastrocnterology 68: 1543-- 1555, 1975.

${ }^{20}$ Berthelot $P$, and D. Dhumeatx. New insights in to the classification and mechanisms of hereditary, chronic. non--haemolytic hyperbilirubinemias. Gut. 19: 478-480, 1978 .

21 Berk $P$. ef ols.: Unconjugated hyperbilirubinemia Annals of lnt. Yed. 82: 552, 1975.

22 Childs $B$. and $V$. Najjar. Famiual nonlemolytic Jaundice with Kernicterus Ped : 18: 369, 1956.

23 Matsuda I et als. Effects of Aspartic Acid and Orotic Acid upon bile serume bilirubin kevels in newborn infants J. of Exp. Med. 90: 133, 1966.

24 Garotti F. Finocchi ef als.: Congenital non hacmolytic jaundice in a 4 year ald girl without disease of the CNS. Itelv. Pacd. Act $24: 399,1969$.

25 Arrowsmith W. ef als.: Comparison of treatment for Congenital Nenatal non haemolytic hyperbilirubjnemia. Arch. Dis. Child. 50: 197, 1975.

26 Blachke IB et ats.: Drugs and the liver Bjochem Pharmacol. 23: 2795 - 2806, 1974.

27 Axias I. Inheritable and Congenital hyperbilirubinemia. New lingl. J. of Med. 285: 1416-1421, 1971 .

${ }^{28}$ Redinger et als: Effect of Phenobarbitone upon bile salt and Pool size. J. C.i. inv. 52:161, 1973.

29 Yaffe, S. Leyy: Enhancement of glucuronide - conjugating capacity in a hyperbilirubinemia infant due to apparent enzyme induction by phenobarbitone. New Eng. J. Med. 275: 1461, 1966.
${ }^{30}$ Gotze, H.D. Sidiropoulos et als. Das Crigler-Najjar Syndrome. Klinische, biochemische, morphologisehe und therapeutische aspekte Helv. Paed. act. 27: $335-351,1977$.

${ }^{31}$ Owens, D., and Sherlock S D Diagnosis of Gilbert's syndrome. Role of reduced caloric intake test. Brit. Med. J. 3: 559-563, 1973.

32 Black M. and Sherlock s. Treatment of Gilbert's syndrome with phenobarbitone. Lancet 1: 1359-1362, 1970.

33 Bepk P.D. et al: A ncw approach to quantitation of the various sources of bilirubin in man. Journal of lab and Сin. Med. 87: 767-780, 1976.

34 Carulli, $N$. Ponz de leon et als.: Alteration of drug metabolism in Gitecrt's syndromc. Gut. 17: $581--587$, 1976.

35 Berthelot P. and D. Dhuncatur. New Insights into the classification and mechanisans of hereditary, chronic, non - haemolytic hyperbiliruhinacmias. Gut. 19: 474-480, 1978.

36 Ben-Ezzer. J. Rimington, C. at als.: Abnornal excretion of the urinary coproporphyrin by patients with Dubin-Johnson syndrome in Isract. Clin. Science. 40: 17. 30, 1971 .

37 Dhumaux, D. and Berthelot, P. Chonic hyperbilirubinemia assuciated with hepatic uptikc and storage impairment. A new syndrome rescmbling that of the mutant southdown sheep. Gastrocnterology 69: 988-993, 1975 .

38 Arias, 1. Transfer of bilirubin from blood to bile. Seminars in Hematology $9: 55-70,1972$ 